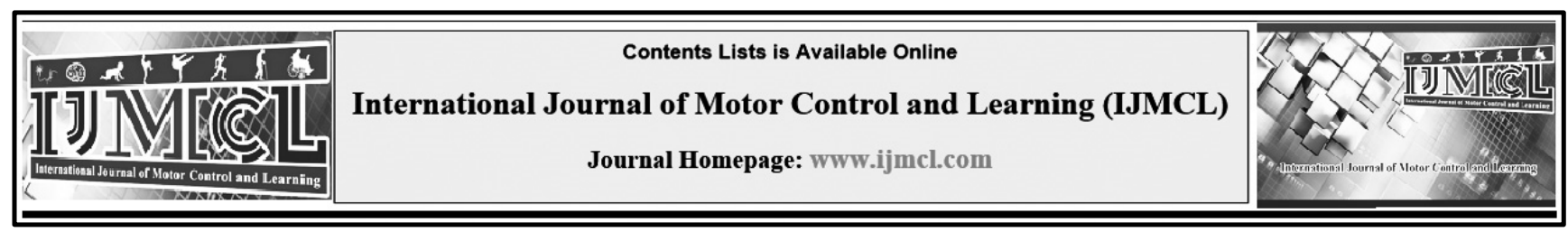

\title{
Effect of Functional Strength Training on Gait Kinematics, Muscle Strength and Static Balance of Young Adults with Down Syndrome
}

\author{
Nassour Ahmadi ${ }^{a *}$, Fatemeh Peyk $^{a}$, Fariborz Hovanloo $^{b}$, Sahel Hemati Garekani $^{c}$ \\ a Department of Motor Behavior, Faculty of Sport Sciences, Shahid Beheshti University, Tehran, Iran \\ ${ }^{\mathrm{b}}$ Department of Sport and Exercise Physiology, Faculty Sport Sciences, Shahid Beheshti University, Tehran, Iran \\ ${ }^{c}$ Department of Psychiatry, University of Social Welfare and Rehabilitation Sciences, Tehran, Iran
}

\begin{tabular}{l}
\hline Keywords \\
\hline Down Syndrome \\
Functional Strength Training \\
Gait Kinematics \\
Isokinetic Peak Torque \\
Static Balance
\end{tabular}

Nassour Ahmadi,

Email: $\underline{\text { n_ahmadi@sbu.ac.ir }}$

Received: 2019/11/25

Accepted: 2020/02/02

Published: 2020/03/03

\begin{abstract}
Background: Atypical gait pattern is one of the most important motor impairments in individuals with Down syndrome.

Objectives: This study aimed to evaluate the effect of functional strength training on gait kinematics, isokinetic peak torque, and static balance of adults with Down syndrome.

Methods: Thirteen men with Down syndrome from Iran Down Syndrome Association participated in this study. The kinematics variable, isokinetic peak torque, and static balance were assessed by motion analysis, isokinetic dynamometer, and Standing Stork Test respectively before and after completing the training study. All subjects were allocated to experimental (Mage $=28.75 \pm 6.1$; $\mathrm{n}=7$ ) and control (Mage $=30 \pm 8.5 ; \mathrm{n}=6$ ) groups according to their desire to participate, or not, in the training program. The exercise protocol consisted of 6week functional strength training (treadmill walking and step up/down training) for the experimental group. One-Way ANCOVA Independent test was employed for data analysis.

Results: The results revealed that the functional strength training improved step length, walking velocity, ankle and knee ROMs (range of motion in sagittal plane), isokinetic peak torque of lower extremities, and static balance. However, no significant difference occurred in step width and hip ROMs.

Conclusions: These findings reinforce previous research showing that taskoriented training with respect to the principle of specificity of training promotes movement patterns in individuals with Down syndrome.
\end{abstract}

\section{Introduction}

Atypical gait pattern is one of the most important motor impairments in individuals with Down syndrome (DS); since it influences the development of cognitive, social, and other complex motor skills such as running and jumping. It is also the most consistent predictor of future falls (El-Meniawy, Kamal, \& Elshemy, 2012; Gontijo et al., 2008). In general, delays in onset of independent walking, premature decline of the gait patterns, and spatiotemporal differences in gait patterns in lifespan make gait patterns unique in individuals with DS (Angulo-Barroso, Burghardt, Lloyd, \& Ulrich, 2008; El-Meniawy et al., 2012; Enkelaar, Smulders, van Schrojenstein Lantman-de Valk, Geurts, \& Weerdesteyn, 2012). Short step length, wide step width, reduced velocity, limited range of motion (ROM) of the lower extremities, and increased gait cadence are some of the 
spatiotemporal parameters that differentiate gait patterns of these populations from that of healthy people (Angulo-Barroso et al., 2008; Enkelaar et al., 2012; Gontijo et al., 2008; Rigoldi, Galli, \& Albertini, 2011).

There is an approach to investigate the underlying causes of the deficits in motor performance of individuals with DS (Almeida, Corcos, \& Latash, 1994). According to this approach, the suboptimal performance of an otherwise intact motor control system is introduced as the reason of the apparent differences in motor performance of individuals with DS from the general population. It also suggests that adequate practice might be able to increase the intensity with which they activate their motor neuron pools, generating more force and providing movement patterns which are similar to those observed in individuals who are neurologically normal (Almeida et al., 1994). Improvement in various movement patterns (kinematics, kinetics, EMG, synergy) have been made in these individuals by physical exercises, which is a solid evidence to support this approach (Amini, Fazel Kalkhoran, Salehi, \& Jazini, 2016; Elshemy, 2013; Lin \& Wuang, 2012; Smith, Stergiou, \& Ulrich, 2011). Consequently, it is likely that appropriate exercises can improve gait patterns in them. However, Masso-Ortigosa et al. (2018) in electromyographic analysis of ankle muscles, in young adults with Down syndrome before and after the implementation of a physical activity program based on dance, observed no significant differences in Down syndrome group after training (Massó-
Ortigosa, Gutiérrez-Vilahú, Costa-Tutusaus, Oviedo, \& Rey-Abella, 2018).

Weakness in both muscle strength and balance which are common in this population are important factors that cause spatiotemporal differences in gait patterns of individuals with DS from the general population. For instance, increases in cocontraction affecting gait kinematics such as ROM are considered a mechanism to compensate muscular weakness in individual with DS (Mirelman, Patritti, Bonato, \& Deutsch, 2010; Rigoldi et al., 2011). Further, lack of balance not only reduces step length and walking velocity but also increases wide step width in these individuals (Mirelman et al., 2010; Rigoldi et al., 2011). Thus, impaired muscle strength and balance are the concerns that must be considered in the process of movement patterns improvement.

Although numerous training programs have been designed to improve muscle strength and balance in these individuals, it has yet to be determined what kinds of strength and balance exercises are appropriate to correct their movement patterns such as walking. Improvement in activities that occur in all planes of motion requires more efficient and effective training than one on isolated muscle and throughout single planes of motion (traditional training) (Clark, Lucett, \& Kirkendall, 2010). Therefore, optimal neuromuscular productivity and performance necessitate dynamic stabilization in other planes plus movement in the single plane in which walking is predominant (Elsawy, 2010). Therefore, it seems that functional training is more appropriate for this purpose 
because not only do they exploit major muscle groups responsible for gait in all three plans of motion, but also improve the specific postural control mechanism (Rigoldi et al., 2011). Considering the principle of specificity, this type of training which replicates natural daily movements or sport performance may be the most effective method at improving muscular fitness (Elsawy, 2010; Weiss et al., 2010). Among functional training programs, functional strength training is the practice of motion against resistance to improve a participant's ability to perform a specific activity and his/her specific muscular fitness (strength and balance) during everyday movements (Elsawy, 2010).

The focus of this paper is on the improvement of gait pattern by functional strength training programs through both movement-based exercise and increased specific muscular fitness. This paper thus aims to determine whether or not functional strength training has any influence on the muscle's strength, balance, and gait kinematics.

\section{Method}

\section{Participants}

A convenience sample of thirteen men with DS (only trisomy 21 form) was recruited through the Down Syndrome Association of Iran (DSAI) to participate in the study. participants were ranging 22-28 years old and were able to follow simple instructions. All participants or their guardians signed a written informed consent form prior to the study conducted at the Exercise Physiology Laboratory of Shahid Beheshti University.
Subjects with associated cardiovascular conditions, blindness, and deafness were excluded.

\section{Protocols}

First, their individual characteristics (height, weight) were measured. Then, they took part in tests including the measurement of gait kinematics, lower extremities isokinetic muscle strength, and static balance at the baseline (week 0). Same measurements were collected immediately after the intervention period (week 6).

All subjects were assigned to either the experimental $(\mathrm{n}=7)$ or the control $(\mathrm{n}=6)$ groups by random blocking based on isokinetic strength test of knee extensor muscles. The control group continued through their usual activities while the experimental group followed the 6-week program of functional strength training consisting of three 60-min sessions per week.

Treadmill walking program and step up/down with some load were considered as the functional strength training exercises in this study due to their similarity in fundamental principles of gait pattern (motor control, biomechanics, and muscle contraction pattern) (Lafferty, 2005; Ulrich, Lloyd, Tiernan, Looper, \& Angulo-Barroso, 2008). Principles of individualization and progressive overload were applied along with principle of specificity training (the main principle of functional training) to improve the effect of exercise. As suggested in previous studies, the intensity of the exercises has been increased individually through gradual progress of medicine ball load, repetition in step-up down, resistance of 
fitness band, and speed of treadmill (Carmeli, Kessel, Coleman, \& Ayalon, 2002; Shields \& Dodd, 2004). Measurement test techniques are described next.

\section{Gait kinematics}

Kinematic data were collected with an eightcamera Osprey Digital Real Time System. Marker displacements followed the Davis protocol (Davis III, Ounpuu, Tyburski, \& Gage, 1991). Participants were instructed to walk barefoot at their selfselected speed along a $4 \mathrm{~m}$ walkway. Six trials were recorded for each subject. Spatiotemporal parameters including step length (SL), step width (SW), and velocity (v); ROM for hip (H-), knee (K) and ankle (A-) in the sagittal (-s) plane were calculated. Kinematic data were filtered with the Butterworth low-pass filter at a cut-off frequency of $10 \mathrm{~Hz}$, established through residual analysis.

\section{The isokinetic peak torque}

The peak torque values of muscles (knee extensor, knee flexors, ankle dorsiflexors, and ankle plantar flexors) were measured using a cybex II isokinetic dynamometer (Biodex, Multi-Joint System, Pro 4, USA). After the warm-up, subjects performed several practice repetitions for each movement at speeds of 60 degrees per second. Finally, they performed two maximal voluntary contractions at a speed of 60 degrees per second, the best of which was chosen for data analysis. All tests were conducted in the sitting position.

\section{Static balance}

Static balance data were collected by Standing Stork Test. This test was carried out three times, and the best result was used for data analysis (Lahtinen, Rintala, \& Malin, 2007).

\section{Treadmill exercise protocol}

The Sample Fitness Treadmill Model JS5000B was used for treadmill exercises. The treadmill training protocol included two 10-minute periods (with"\%0" incline). There were 4 color fitness bands (violet, red, yellow, and blue) to increase the intensity of resistance. These bands had been designed such that one side was connected to the anterior part of the shoes and the other side was attached to a bar placed at a distance of 1 meter from the end of the treadmill. All participants started the treadmill training with zero intensity (band resistance) and walked as fast as they could comfortably tolerate. Initial predetermined speed ranged from $2 \mathrm{~km} / \mathrm{h}$ to $3 \mathrm{~km} / \mathrm{h}$ for participants according to their physical capability. During the training sessions, the treadmill speed for all participants was gradually increased up to $3 \mathrm{~km} / \mathrm{h}$. When the subjects were able to accomplish comfortably 2 sets of 10-minute at $3 \mathrm{~km} / \mathrm{h}$ with zero intensity, the band intensity was increased. This procedure was continued in the new intensity level.

\section{The step up/down training}

This exercise was carried out with medicine balls which were 2, 3, and $4 \mathrm{~kg}$. Participants ascended the step (40-cm high) with the right foot 
and then descended with the same one. They should do plantar flexion when placed on the ground for strengthening plantar flexors which are important muscles during walking. After completing this section, they did the same training with the left foot. In order to enhance motivation of the participants, we installed a hoop proportionate with their height in front of them and they were asked to drop medicine ball into the hoop with plantar flexion. All participants started the training with 2kilogram- weight medicine ball and the protocol included 2 sets of between 8 to 14 repetitions (range was determined based on fatigue threshold in a session individually). Repetitions were gradually increased to 14 ; when the participants were able to complete 2 sets of 14 repetitions for 2 consecutive sessions using the proper lifting technique, the load was increased to the next intensity. The time period of rest was twice as long as the training period. Some encouragements were offered such as conversation about their interests (e.g. movies, athletes), music, verbal encouragement, and colorful equipment to motivate the individuals to participate actively and to maximize the training effects.

\section{Statistical analysis}

All statistical analyses were done using SPSS v18.0. Means and standard deviations were calculated for all variables. Because of inequality of pre-tests $\left(\mathrm{p}_{\mathrm{s}}<.05\right)$, one-way ANOVA was used when the regression line is homogeneous. However, if the homogeneity of regression line was not seen, at first the difference between post- and pre-test was calculated, and then independent $t$ test was utilized to compare control and experimental groups. Statistical significance was set at $\mathrm{p} \leq 0.05$.

\section{Results}

Participants completed all testing and training with no adverse events. There were no differences between groups for any demographic variables (Table 1).

Table 1: Demographics of Data for Experimental and Control Groups.

\begin{tabular}{lcc}
\hline Characteristics & Experimental & Control \\
\hline Gender & Male $(n=7)$ & Male $(n=6)$ \\
Age (years) & $28.75 \pm 6.1$ & $30 \pm 8.5$ \\
IQ & $63.85 \pm 5.2$ & $62 \pm 8.78$ \\
Weight $(\mathrm{kg})$ & $66.62 \pm 11.17$ & $66.05 \pm 7.2$ \\
Height $(\mathrm{cm})$ & $160.72 \pm 7.37$ & $158.05 \pm 7.2$
\end{tabular}

\section{Kinematics}

The experimental group showed a significant increase in step Length $\left(\mathrm{F}_{1,11}=63.23 ; \mathrm{P}=0: 005\right)$, walking velocity $(\mathrm{t}=5.09 ; \mathrm{P}=0.005), \operatorname{AROM}(\mathrm{t}=$ 5.06; $\mathrm{P}=0.005)$, and $\operatorname{KROM}\left(\mathrm{F}_{1,11}=7.39 ; \mathrm{P}=0.02\right)$ over the 6-week training period compared to control group, whereas there were no significant differences in step width $\left(\mathrm{F}_{1,11}=0.03 ; \mathrm{P}=0.85\right)$ and $\operatorname{HROM}\left(\mathrm{F}_{1,11}=1.94 ; \mathrm{P}=0.19\right)$ between groups as represented in Table 2.

\section{Isokinetic Peak Torque}

The isokinetic peak torque of knee flexors $\left(F_{1}\right.$, $11=11.84 ; \mathrm{P}=0.006)$, knee extensors $\left(\mathrm{F}_{1,11}=\right.$ 11.65; $\mathrm{P}=0: 007)$, ankle dorsi flexors $\left(\mathrm{F}_{1,11}=6.34\right.$; $\mathrm{P}=0.03)$, and ankle plantar flexors $\left(\mathrm{F}_{1,11}=37.93\right.$; $\mathrm{P}=0.005)$ was remarkably improved for the 
experimental group compared to the control group as showed in Table 3.

\section{Static Balance}

Likewise, the Stork Balance Stand Test scores balance score $\left(\mathrm{F}_{1,11}=14.49 ; \mathrm{P}=0.003\right)$ increased notably from $6.19 \mathrm{~s}$ to $13.06 \mathrm{~s}$ in the experimental group as documented in Table 3, whereas no significant differences were found for the control group.

Table 2: Kinematic Parameter Results for Experimental and Control Groups.

\begin{tabular}{ccccc}
\hline \multicolumn{4}{c}{ Experimental } & \multicolumn{2}{c}{ Control } \\
Variables & Pre & Post & Pre & Post \\
\hline SL $(\mathrm{mm})$ & $507.43 \pm 55.2^{*}$ & $567.45 \pm 48.22$ & $488.35 \pm 45.20$ & $492.31 \pm 35.76$ \\
SLV $(\mathrm{mm})$ & $32.66 \pm 6.9^{*}$ & $25.24 \pm 3.6$ & $35.49 \pm 12.99$ & $36.12 \pm 13.63$ \\
SW $(\mathrm{mm})$ & $145.58 \pm 9.62$ & $142.24 \pm 3.4$ & $145.7 \pm 9.85$ & $141.93 \pm 13.64$ \\
SWV & $17.77 \pm 4.2^{*}$ & $15.45 \pm 3.88$ & $17.65 \pm 2.3$ & $17.74 \pm 2.79$ \\
V $(\mathrm{m} / \mathrm{s})$ & $0.79 \pm 0.1^{*}$ & $1.21 \pm 0.11$ & $0.81 \pm 0.13$ & $0.81 \pm 0.15$ \\
AROMs & $22.4 \pm 2.8^{*}$ & $26.82 \pm 1.86$ & $20.91 \pm 2.37$ & $20.55 \pm 2.5$ \\
KROMs & $49.81 \pm 4.57^{*}$ & $55.97 \pm 1.61$ & $49 . \pm 6.2$ & $49.17 \pm 5.7$ \\
HROMs & $39.17 \pm 1.6$ & $41.3 \pm 2.3$ & $49 . \pm 6.2$ & $38.81 \pm 3.6$ \\
\hline${ }^{*} p<0.05$ pre-test vs. post-test in the experimental group. &
\end{tabular}

Table 3: Physical Variable Results for Experimental and control Groups.

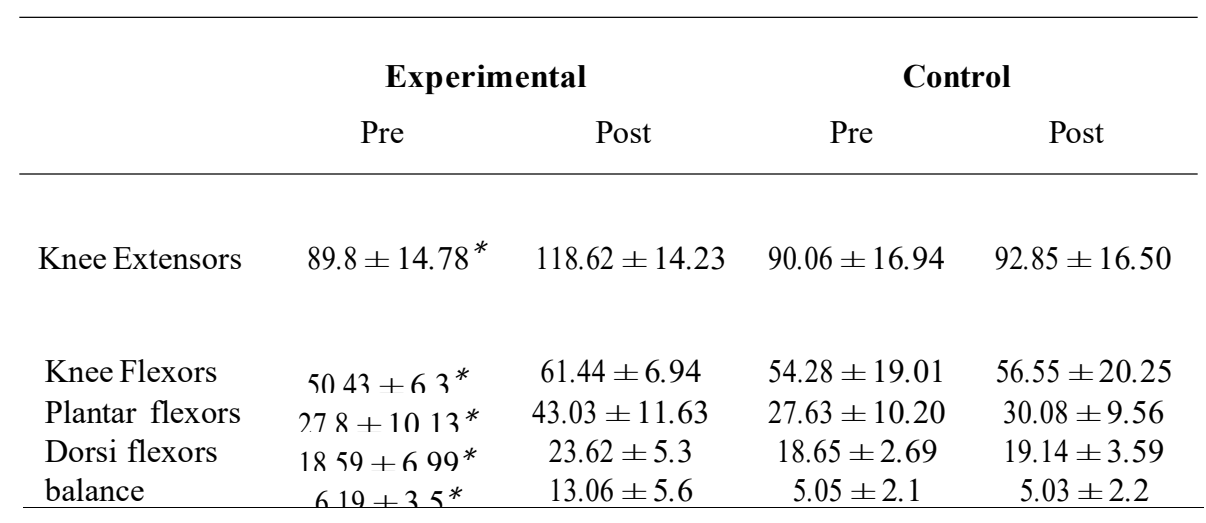

${ }^{*} p<0.05$ pre-test vs. post-test in the experimental group.

\section{Discussion}

Results from the present study demonstrated that a 6-week functional strength training improved step length, walking velocity, AROMs, and KROMs in subjects who participated in the program. Further, there was significant improvement in terms of isokinetic peak torque of lower extremities and static balance for them.
However, no remarkable difference was observed in step width and HROMs. The findings of this study validate that task-oriented training considering the principle of specificity of training promotes movement patterns in individuals with DS, which has been reported in the literature (Almeida et al., 1994; Lafferty, 2005; Ulrich et al., 2008). They also verify that adults with DS were 
able to improve their muscle strength of lower extremities and balance notably through participation in this training program (Carmeli et al., 2002; Shields \& Dodd, 2004; Tsimaras \& Fotiadou, 2004).

The improvement in muscle strength and balance may be significantly pertinent to adults with DS since their daily activities and workrelated skills require lower limbs movement and balance. Further, both muscle strength and balance are subcategories of fitness which are important to general health (Lin \& Wuang, 2012; Tsimaras \& Fotiadou, 2004). As discussed before, impaired muscle strength and balance impact spatiotemporal parameters of gait, especially ROM, in individuals with DS (Mirelman et al., 2010; Rigoldi et al., 2011). Therefore, improvements in balance and strength of major muscle groups responsible for gait are likely to be the reason for changes in spatiotemporal parameters of gait (Anwer, Equebal, Kumar, \& Nezamuddin, 2012; Rigoldi et al., 2011). Thus, increase in ankle dorsal and plantar flexors strength and improved balance are likely to increase the AROMs. Ankle plantar flexor muscles are of the most important muscles involved in walking whose insufficient activity not only reduces the AROMs, but also can result in poor control of the tibia during stance phase; especially push-off. In this situation, the position of the knee during mid-stance (with the knee collapsing into flexion) is the most frequently adopted pattern for compensation. However, improved tibial control causes less residual flexion and better extension with increased strength. That is, distal control achieved through training could result in improved proximal control (Mirelman et al., 2010). In general, the changes in KROMs during stance are likely to be associated with an increase in ankle control that allowed for better tibial progression in addition to increased strength and balance (Galli et al., 2010; Mirelman et al., 2010; Rigoldi et al., 2011). No specific changes were observed in HROMs of adults with DS in this study, which may be due to several reasons. According to previous researches, it is possible that motion control at hip level in such individuals is as high as that of normal people. That is, HROMs was in fact characterized by values somewhat closer to optimal walking performance (Galli, Rigoldi, Brunner, Virji-Babul, \& Giorgio, 2008). Furthermore, it may be linked to the different anatomical configuration of their pelvic girdle called "Mongol Pelvis" which is a permanent trait (Elshemy, 2013).

Step length was the other kinematic parameter which increased significantly after six weeks. The poor angular impulse in the push off phase affects step length (Gupta \& Mehta, 2009; Hass, Buckley, Pitsikoulis, \& Barthelemy, 2012; TeixeiraSalmela, Nadeau, Mcbride, \& Olney, 2001). An improvement in the major muscle torque, AROMs, or both increases the angular impulse and possibly leads to an increased step length (Gupta \& Mehta, 2009; Hass et al., 2012; Teixeira-Salmela et al., 2001). According to the results, balance improvement is the other factor which can improve step length because unsteady gait increases cautiousness during walking and may lead to short 
strides. This cautiousness can be reduced slightly by increasing the balance, which projects their center of mass forward and hence improves gait by increasing step length (Bello et al., 2013; Weiss et al., 2010).

A notable increase in walking velocity is the other finding of the study. There is a strong positive relationship between velocity and step length (Elshemy, 2013). It can be concluded that the increase in walking velocity is associated with an increase in stride length. Further, peak ankle plantar flexion (at or before toe-off) facilitates a mechanical energy transfer from gravitational potential energy to forward kinetic energy. It can shift the location of the center of mass from the ipsilateral leg to the contralateral leg, producing a higher net mechanical output to the swing phase and yielding an increase in walking velocity $(\mathrm{Wu}$, Looper, Ulrich, \& Angulo-Barroso, 2010).

The step width parameter remained unchanged. Increasing step width probably is not a preferred gait strategy among young, active adults with DS. Instead, they appear to use other strategies such as decreased velocity and short step length to partially compensate for lesser stability (Agiovlasitis, Pavol, McCubbin, \& Yun; Enkelaar et al., 2012). Accordingly, participants in this study may not use step width as a strategy to make up for instability because other strategies (velocity and step length) improved as a result of functional strength training while step width is remained unchanged (Agiovlasitis et al.).

In addition to the factors proposed for gait pattern improvement, it seems that the senses and neural circuits related to walking may be changed as a result of functional strength training (Rodacki, Souza, Ugrinowitsch, Cristopoliski, \& Fowler, 2009). Increase in ROMs not only boosts force generation, but also reduces the impact forces at heel strike. It may help to reduce head acceleration during progression and may provide a facilitated stabilization of the visual platform and fewer disturbances over the vestibular apparatus (Enkelaar et al., 2012; Rigoldi et al., 2011). Then, increases in force absorption can enhance afferent sensory related to gait which is impaired in subjects with DS (Elshemy, 2013; Rigoldi et al., 2011). Also, other mechanisms have been proposed to explain the improvement in gait induced by treadmill training. For example, the treadmill may provide proprioceptive signals, trigger intact circuits, strengthen and stabilize the neural network involved in producing walking (Bello et al., 2013; El-Meniawy et al., 2012; Ulrich et al., 2008).

The functional training implemented for this study was feasible for adults with DS. It might be expected that adults with DS have difficulty taking part in or being motivated to continue with a functional training. Compliance with the program was excellent, with participants attending $92 \%$ of all training sessions. There were also no withdrawals from the study, indicating that the functional training program was an acceptable form of exercise to the participants. Additionally, the implemented exercise training program seemed to be rather safe since no major adverse events were reported by the participants. Then, interaction of a motivational environment and efficient and 
adequate training overcomes the avoidant learning styles adopted by individual with DS in this study (Almeida et al., 1994). This approach influences the self-efficacy of them by maintaining motivation and adherence to task (Lin \& Wuang, 2012; Shields $\&$ Dodd, 2004). This finding also challenges the viewpoint that individuals with DS should not take part in physical activities because of their health concerns (Lin \& Wuang, 2012).

One of the most important limitation of this study was speed of walking. In this study, subjects were asked to walk with preferred speed. Increasing in speed may affect other kinematic variables because of need to muscular strength.

Overall, the results of this study confirmed previous findings that lack of opportunity to practice and their rather inactive lifestyle may be a major cause of the deficits in motor performance of individuals with DS (Almeida et al., 1994; Enkelaar et al., 2012). Also, these findings supported previous researches that task-oriented training with respect to the principle of specificity of training promote movement patterns in individuals with DS.

\section{Conclusions}

Six weeks of the functional training implemented for this study improved the gait pattern and decreased abnormalities of gait pattern, the most consistent predictors of future fall. These findings reinforced previous research which believes that task-oriented training with respect to the principle of specificity of training promotes movement patterns in individuals with DS. It also verified that adults with DS improved their muscle strength of lower extremities and balance notably through participation in this training program.

\section{Acknowledgement}

We thank Iran Down Syndrome Association especially the parents and individuals with DS who participated in this study for their commitment to all scheduled evaluations.

\section{References}

1. Agiovlasitis, S., Pavol, M. J., McCubbin, J. A., \& Yun, J. Effects of down syndrome on mediolateral motion during walking at different speeds.

2. Almeida, G. L., Corcos, D. M., \& Latash, M. L. (1994). Practice and transfer effects during fast single-joint elbow movements in individuals with Down syndrome. Physical Therapy, 74(11), 1000-1012.

3. Amini, H. A., Fazel Kalkhoran, J., Salehi, M., \& Jazini, F. (2016). Effect of backward walking training on improves postural stability in children with down syndrome. International Journal of Pediatrics, 4(7), 2171-2181.

4. Angulo-Barroso, R., Burghardt, A. R., Lloyd, M., \& Ulrich, D. A. (2008). Physical activity in infants with Down syndrome receiving a treadmill intervention. Infant Behavior and Development, 31(2), 255-269.

5. Anwer, S., Equebal, A., Kumar, R., \& Nezamuddin, M. (2012). Effect of closed kinematic chain exercises in improving gait and function in cerebral palsy: a case report. International Journal of Therapy and Rehabilitation, 19(7), 414-418.

6. Bello, O., Sánchez, J. A., Lopez-Alonso, V., Márquez, G., Morenilla, L., Castro, X., . . . Fernández-del-Olmo, M. (2013). The effects of treadmill or overground walking training program on gait in Parkinson's disease. Gait \& posture, 38(4), 590-595.

7. Carmeli, E., Kessel, S., Coleman, R., \& Ayalon, M. (2002). Effects of a treadmill walking program on muscle strength and balance in elderly people with Down syndrome. The Journals of Gerontology Series A: Biological Sciences and Medical Sciences, 57(2), M106M110.

8. Clark, M., Lucett, S., \& Kirkendall, D. T. (2010). NASM's essentials of sports performance training: Lippincott Williams \& Wilkins.

9. Davis III, R. B., Ounpuu, S., Tyburski, D., \& Gage, J. R. (1991). A gait analysis data collection and reduction technique. Human movement science, 10(5), 575-587.

10. El-Meniawy, G. H., Kamal, H. M., \& Elshemy, S. A. (2012). Role of treadmill training versus suspension therapy on balance in children with Down syndrome. 
Egyptian Journal of Medical Human Genetics, 13(1), 3743.

11. Elsawy, G. (2010). Effect of Functional Strength Training on Certain Physical Variables and Kick of Twimeo Chagi among Young Taekwondo Players. World J Sport Sci, 3, 683-686.

12. Elshemy, S. A. (2013). Comparative study: Parameters of gait in Down syndrome versus matched obese and healthy children. Egyptian Journal of Medical Human Genetics, 14(3), 285-291.

13. Enkelaar, L., Smulders, E., van Schrojenstein Lantmande Valk, H., Geurts, A. C., \& Weerdesteyn, V. (2012). A review of balance and gait capacities in relation to falls in persons with intellectual disability. Research in Developmental Disabilities, 33(1), 291-306.

14. Galli, M., Cimolin, V., Patti, P., Ferrario, D., Heaney, G., Albertini, G., \& Freedland, R. (2010). Quantifying established clinical assessment measures using 3Dmovement analysis in individuals with Down syndrome. Disability and rehabilitation, 32(21), 1768-1774.

15. Galli, M., Rigoldi, C., Brunner, R., Virji-Babul, N., \& Giorgio, A. (2008). Joint stiffness and gait pattern evaluation in children with Down syndrome. Gait \& posture, 28(3), 502-506.

16. Gontijo, A., Mancini, M., Silva, P., Chagas, P., Sampaio, R., Luz, R., \& Fonseca, S. (2008). Changes in lower limb co-contraction and stiffness by toddlers with Down syndrome and toddlers with typical development during the acquisition of independent gait. Human movement science, 27(4), 610-621.

17. Gupta, N., \& Mehta, R. (2009). Comparison of gait performance of spinal cord injury subjects: body weight supported treadmill training versus over ground gait training. Apollo Medicine, 6(1), 21-27.

18. Hass, C. J., Buckley, T. A., Pitsikoulis, C., \& Barthelemy, E. J. (2012). Progressive resistance training improves gait initiation in individuals with Parkinson's disease. Gait \& posture, 35(4), 669-673.

19. Lafferty, M. E. (2005). A stair-walking intervention strategy for children with Down's Syndrome. Journal of Bodywork and Movement Therapies, 9(1), 65-74.

20. Lahtinen, U., Rintala, P., \& Malin, A. (2007). Physical performance of individuals with intellectual disability: A 30-year follow-up. Adapted Physical Activity Quarterly, 24(2), 125-143.

21. Lin, H.-C., \& Wuang, Y.-P. (2012). Strength and agility training in adolescents with Down syndrome: a randomized controlled trial. Research in Developmental Disabilities, 33(6), 2236-2244.

22. Massó-Ortigosa, N., Gutiérrez-Vilahú, L., CostaTutusaus, L., Oviedo, G. R., \& Rey-Abella, F. (2018). Electromyographic analysis of ankle muscles in young adults with Down syndrome before and after the implementation of a physical activity programme based on dance. Apunts. Medicina de l'Esport, 53(198), 63-73.

23. Mirelman, A., Patritti, B. L., Bonato, P., \& Deutsch, J. E. (2010). Effects of virtual reality training on gait biomechanics of individuals post-stroke. Gait \& posture, 31(4), 433-437.

24. Rigoldi, C., Galli, M., \& Albertini, G. (2011). Gait development during lifespan in subjects with Down syndrome. Research in Developmental Disabilities, 32(1), 158-163.

25. Rodacki, A. L., Souza, R. M., Ugrinowitsch, C., Cristopoliski, F., \& Fowler, N. E. (2009). Transient effects of stretching exercises on gait parameters of elderly women. Manual therapy, 14(2), 167-172.

26. Shields, N., \& Dodd, K. (2004). A systematic review on the effects of exercise programmes designed to improve strength for people with Down syndrome. Physical Therapy Reviews, 9(2), 109-115.

27. Smith, B. A., Stergiou, N., \& Ulrich, B. D. (2011). Patterns of gait variability across the lifespan in persons with and without down syndrome. Journal of neurologic physical therapy: JNPT, 35(4), 170.

28. Teixeira-Salmela, L. F., Nadeau, S., Mcbride, I., \& Olney, S. J. (2001). Effects of muscle strengthening and physical conditioning training on temporal, kinematic and kinetic variables during gait in chronic stroke survivors. Journal of rehabilitation medicine, 33(2), 5360.

29. Tsimaras, V. K., \& Fotiadou, E. G. (2004). Effect of training on the muscle strength and dynamic balance ability of adults with down syndrome. Journal of Strength and Conditioning Research, 18(2), 343-347.

30. Ulrich, D. A., Lloyd, M. C., Tiernan, C. W., Looper, J. E., \& Angulo-Barroso, R. M. (2008). Effects of intensity of treadmill training on developmental outcomes and stepping in infants with Down syndrome: a randomized trial. Physical Therapy, 88(1), 114-122.

31. Weiss, T., Kreitinger, J., Wilde, H., Wiora, C., Steege, M., Dalleck, L., \& Janot, J. (2010). Effect of functional resistance training on muscular fitness outcomes in young adults. Journal of Exercise Science \& Fitness, 8(2), 113-122.

32. Wu, J., Looper, J., Ulrich, D. A., \& Angulo-Barroso, R. M. (2010). Effects of various treadmill interventions on the development of joint kinematics in infants with Down syndrome. Physical Therapy, 90(9), 1265-1276. 